\title{
Emerging role of sphingosine-I-phosphate signaling in head and neck squamous cell carcinoma
}

This article was published in the following Dove Press journal:

OncoTargets and Therapy

31 May 2016

Number of times this article has been viewed

\author{
Rajeev Nema' \\ Supriya Vishwakarma' \\ Rahul Agarwal ${ }^{2}$ \\ Rajendra Kumar Panday ${ }^{3}$ \\ Ashok Kumar' \\ 'Department of Biochemistry, All \\ India Institute of Medical Sciences \\ Bhopal, Bhopal, 'Jawaharlal Nehru \\ Cancer Hospital \& Research Centre, \\ ${ }^{3}$ Navodaya Cancer Hospital, Indrapuri, \\ Bhopal, India
}

\begin{abstract}
Head and neck squamous cell carcinoma (HNSCC) is the sixth most frequent cancer type, with an annual incidence of approximately half a million people worldwide. It has a high recurrence rate and an extremely low survival rate. This is due to limited availability of effective therapies to reduce the rate of recurrence, resulting in high morbidity and mortality of patients with advanced stages of the disease. HNSCC often develops resistance to chemotherapy and targeted drug therapy. Thus, to overcome the problem of drug resistance, there is a need to explore novel drug targets. Sphingosine-1-phosphate (S1P) is a bioactive sphingolipid involved in inflammation, tumor progression, and angiogenesis. S1P is synthesized intracellularly by two sphingosine kinases (SphKs). It can be exported to the extracellular space, where it can activate a family of G-protein-coupled receptors. Alternatively, S1P can act as an intracellular second messenger. SphK1 regulates tumor progression, invasion, metastasis, and chemoresistance in HNSCC. SphK1 expression is highly elevated in advanced stage HNSCC tumors and correlates with poor survival. In this article, we review current knowledge regarding the role of S1P receptors and enzymes of S1P metabolism in HNSCC carcinogenesis. Furthermore, we summarize the current perspectives on therapeutic approaches for targeting S1P pathway for treating HNSCC.

Keywords: head and neck squamous cell carcinoma, HNSCC, oral squamous cell carcinoma, OSCC, sphingosine-1-phosphate, S1P, sphingosine kinase 1, SphK1, S1P receptor, sphingolipid
\end{abstract}

\section{Introduction}

Head and neck cancer (HNC) is a heterogeneous disease that can arise from cells in the oral and nasal cavity, postnasal spaces (sinuses), lips, salivary glands, pharynx (hypopharynx, oropharynx, and nasopharynx), and larynx and hence can produce tumors at any of these sites. ${ }^{1}$ More than $90 \%$ of HNCs are squamous cell carcinomas and are referred to as head and neck squamous cell carcinoma (HNSCC). ${ }^{1} \mathrm{HNSCC}$ is the sixth most common cancer type and the eighth most frequent cause of cancerrelated deaths worldwide. ${ }^{2-4}$ Squamous cell carcinomas arising in oral cavity are termed as oral squamous cell carcinoma (OSCC). In the Indian subcontinent, OSCC is the most common cancer in men, accounting for one-fifth of all cancers. ${ }^{5}$ HNSCC patients have an average 5-year survival rate of $60 \%$. Despite an expansion of therapeutic strategies for HNSCC over the past three decades, the survival rate for afflicted patients has not increased.

The current treatment for HNSCC usually involves chemotherapy, radiotherapy, or a combination of chemotherapy and radiation, interventions that carry considerable side effects. Molecular targeted therapies offer new treatment options even for heavily pretreated and seriously ill patients who are unable to tolerate chemotherapy or radiation therapy. For example, epidermal growth factor receptor (EGFR)-targeted therapies inhibit cellular proliferation, survival, invasion, and angiogenesis and act synergistically
Correspondence: Ashok Kumar Department of Biochemistry, All India Institute of Medical Sciences Bhopal, Saket Nagar, Bhopal 462020, Madhya Pradesh, India

Tel +9l 7552672319

Email ashok.biochemistry@aiimsbhopal. edu.in 
with chemoradiation combination therapies. ${ }^{6}$ Cetuximab, a chimeric humanized monoclonal antibody against EGFR, is currently approved in the USA and Europe as a monotherapy for patients with platinum-refractory recurrent disease ${ }^{7}$ and as a first-line therapy in combination with platinum-based drugs, 5-fluorouracil or docetaxel, for patients with recurrent or metastatic disease. ${ }^{8,9}$ Several monoclonal antibodies and tyrosine kinase inhibitors are now in Phase III clinical trials for the treatment of patients with HNSCC. ${ }^{9}$ Despite clinical gains arising from the use of cetuximab, both intrinsic resistance and the development of acquired resistance are well-recognized phenomena in HNSCC. ${ }^{10,11}$ Identifying novel mechanisms of carcinogenesis operating in HNSCC could lead to the development of targeted therapy combinations that reduce the occurrence of drug resistance, have less toxicity, and achieve better overall efficacy.

Sphingosine-1-phosphate (S1P) is a biologically active signaling lipid that exerts many of its actions by binding to a family of G-protein-coupled receptors known as S1P receptors (S1PRs). ${ }^{12}$ It regulates several diverse cellular and physiological processes, including cell growth and survival, lymphocyte trafficking, vascular development, and inflammation. ${ }^{13} \mathrm{~S} 1 \mathrm{P}$ signaling is implicated in the processes of carcinogenesis, cancer progression, and drug and radiation resistance. ${ }^{14,15}$ It promotes cancer by inhibiting apoptosis and by enhancing proliferation, transformation, angiogenesis, and inflammation. ${ }^{14,16,17}$ Sphingosine kinase 1 (SphK1), a key enzyme involved in S1P synthesis, promotes tumor progression, invasion, metastasis, and chemoresistance in HNSCC, and its expression level correlates with patient survival. In the present article, we reviewed recent advances in understanding the impact of S1P signaling in HNSCC carcinogenesis and summarized the various pharmaceutical strategies targeting S1P pathway that are being developed for the treatment of HNSCC.

\section{Molecular basis of HNSCC}

The initiation and progression of HNC is a complex multistep process that entails a progressive acquisition of genetic and epigenetic alterations. It has been proposed that OSCC starts with the transformation of a limited number of normal keratinocytes. This transformation may be brought about by epigenetic and cytogenetic changes that affect cell-cycle progression, DNA repair mechanisms, differentiation, and/or apoptosis. ${ }^{18}$ The TP53 (p53 encoding gene) and retinoblastoma $(\mathrm{Rb})$ pathways are almost universally disrupted in HNSCCs, indicating the importance of these pathways in HNSCC tumorigenesis. ${ }^{19}$ In all, $>50 \%$ of HNSCCs harbor inactivating TP53 gene mutations and $>50 \%$ demonstrate chromosomal loss at $17 \mathrm{p}$ - the site where the TP53 gene resides. The progression from the $\mathrm{G} 1$ phase to $\mathrm{S}$ phase is a critical checkpoint in protecting the cells from abnormal replication, and a key regulator of this process is the cyclindependent kinases (CDK) 4/6-INK4-Rb pathway. Activation of the CDK 4/6 complex contributes to the hyperphosphorylation of the $\mathrm{Rb}$ protein, which causes inactivation of its growth-inhibitory function by decoupling it from E2F transcription factors. The newly released E2F transcription factors allow the transcription of genes promoting entry into the $\mathrm{S}$ phase and thus cell-cycle progression. The most frequently mutated component of the Rb pathway in HNSCC is the $p 16 I N K 4 A$ tumor suppressor gene. Its gene product prevents cells from entering the cell cycle by inhibiting CDK4 and CDK6. Inactivation of $p 16 I N K 4 A$ can occur via promoter hypermethylation, gene mutation, or deletion, the latter being recognized genetically as a loss of heterozygosity (LOH). Indeed, $\mathrm{LOH}$ at the chromosomal region 9p21 (where p16INK4A resides) occurs in up to $80 \%$ of HNSCC. In addition to the inactivation of $p 16 I N K 4 A$ and TP53, mutations in other genes that regulate cell proliferation, including CDKN2A, PTEN, PIK3CA, and HRAS, have been implicated in HNSCC. ${ }^{20}$ Whole-exome sequencing data have also shown that $30 \%$ of all HNSCC cases harbored mutations in genes that regulate squamous differentiation (eg, NOTCH1, IRF6, and TP63), implicating its dysregulation as a major driver of HNSCC carcinogenesis. ${ }^{21}$

In HNSCC, at least two genetic subclasses can be distinguished: human papillomavirus (HPV)-positive and HPV-negative tumors. ${ }^{20}$ The presence of HPV is a prognostic biomarker associated with better outcome in locally advanced oropharyngeal cancers, with a 40\%-80\% cure rate. HPV type 16 is a strictly epitheliotropic, circular doublestranded DNA virus that is known to cause cervical cancer in women. ${ }^{22}$ The HPV virus produces two oncoproteins, E6 and E7, which inactivate p53 and Rb, respectively, thereby promoting unrestrained cell-cycle progression in the infected cells. This is considered the driving force of HPV-mediated carcinogenesis. Mutations in TP53 occur in $45 \%-70 \%$ of HNSCC. ${ }^{23}$ However, HNSCC tumors that are HPV positive, meaning they express the E6- and E7-oncogene transcripts, are typically wild type for $T P 53,{ }^{20}$ and patients with this category of tumors have favorable prognosis.

TP53 mutations are frequently accompanied by loss of chromosome $3 p$ and are associated with advanced clinical stage in HNSCC patients. ${ }^{24}$ A mitogenic signaling pathway that includes EGFR, HRAS, and phosphatidylinositol 3-kinase 
(PI3K) has been shown to be altered in $>60 \%$ of OSCC, and EGFR is overexpressed in $>90 \%$ of HNSCC. ${ }^{25,26}$ In HNSCC, constitutive activation of EGFR can occur via overexpression of EGFR ligands, mutation or amplification of EGFR, or by activation of other receptors or nonreceptor tyrosine kinases. ${ }^{27} \mathrm{LOH}$ at chromosomes $3 \mathrm{p}, 9 \mathrm{p}$, and $17 \mathrm{p}$ seems to occur in dysplasia, apparently reflecting early carcinogenesis, whereas other alterations at chromosomes 11q, 4q, and 8 were typically present in carcinomas, probably corresponding to a relatively late phase in carcinogenesis. ${ }^{28}$

\section{SIP signaling}

$\mathrm{S} 1 \mathrm{P}$ is a potent signaling molecule that is present in blood, lymph, and most tissues. It mediates pleiotropic biological functions, including hematopoietic cell trafficking, T-cell differentiation, immunological and allergic responses, inflammation, angiogenesis, vascular integrity, and muscle regeneration. ${ }^{12,29,30}$ At the cellular level, S1P regulates apoptosis, cell proliferation, survival, migration, invasion, differentiation, and intracellular architecture. ${ }^{12}$ It mediates most of its functions by binding to a family of G-protein-coupled receptors, formerly termed endothelial differentiation genes, but now these receptors are known as S1PRs 1-5.29,31,32

In addition to mediating effects via extracellular receptormediated signaling, S1P also acts intracellularly and independent of its receptors. Only few intracellular targets of $\mathrm{S} 1 \mathrm{P}$ have been identified. For example, S1P directly binds to TNF receptor-associated factor 2, an E3 ubiquitin ligase that is a key component of the nuclear factor $(\mathrm{NF})-\mathrm{\kappa B}$ pathway. Both, SphK1 and the production of S1P are necessary for TNF-mediated NF- $\kappa$ B activation. ${ }^{33}$ S1P formed by SphK2 in the nucleus directly binds to histone deacetylase (HDAC) and regulates gene expression. ${ }^{34}$ Moreover, it has also been shown that S1P binds and modulates the activity of $\beta$ amyloid precursor protein cleaving enzyme 1 in vitro. ${ }^{35}$ S1P signaling has a significant impact on the processes of carcinogenesis and drug- and radiation-resistance patterns. Many components of the S1P signaling pathway, including SphKs, S1P lyase, and S1PR, have been mechanistically linked with cancer cell aggressiveness, invasion, metastasis, and chemoresistance. ${ }^{14,30}$

\section{Circulating SIP in cancer}

In the blood of healthy subjects, S1P concentrations vary in the range of $0.2-1.0 \mu \mathrm{M}$ and depend upon age and sex. Erythrocytes and platelets are rich sources of plasma S1P, as both of these cell types are exposed to circulating sphingosine, contain SphK activity, and lack most of the S1P-degrading enzymes. ${ }^{29,36}$ In blood, S1P circulates in the high-density lipoprotein (HDL)-bound (65\%) and albumin-bound (35\%) forms. ${ }^{37}$ Further, it has been shown that apolipoprotein M, a lipocalin that resides mainly in the plasma HDL fraction, acts as a carrier of S1P in blood. Furthermore, the HDLassociated apolipoprotein $\mathrm{M}-\mathrm{S} 1 \mathrm{P}$ complex mediates vasoprotective actions on the endothelium. ${ }^{38}$ HDL-bound S1P restrains lymphopoiesis and neuroinflammation by activating S1PR1 on bone marrow lymphocyte progenitors. ${ }^{39}$ Several investigators have explored the potential of plasma S1P to serve as a biomarker for the detection of cancer. In ovarian cancer patients, plasma S1P levels were almost twice as high as those of healthy controls. ${ }^{40}$ In a separate study, elevated plasma S1P levels have been associated with the increased risk of developing lung cancer. ${ }^{41}$ In contrast, in prostate cancer patients, plasma S1P levels were lower than in agematched controls. ${ }^{42}$ Comparisons of plasma S1P levels in HNSCC patients and age- or sex-matched controls have not been reported to date.

\section{SIP metabolizing enzymes in HNSCC}

Considering the pleiotropic biological functions mediated by $\mathrm{S} 1 \mathrm{P}$, it is not surprising that its cellular and circulating levels are tightly regulated by several enzymes ${ }^{14-16}$ (Figure 1). Two highly homologous SphKs, known as SphK1 and SphK2, catalyze the phosphorylation of sphingosine to form S1P. ${ }^{12}$ Sphingosine is generated via the deacylation of ceramide, which in turn is produced by the hydrolysis of sphingomyelin present in the outer leaflet of the plasma membrane. ${ }^{16,43}$ Two S1P phosphatases (SGPPs) known as SGPP1 and SGPP2, located in the endoplasmic reticulum, reversibly convert S1P back to sphingosine. ${ }^{44,45}$ In addition to SGPPs, there are three plasma membrane-bound phosphatases known as lipid phosphate phosphatases 1, 2, and 3 (LPP1-3) that can dephosphorylate a broad range of lipid phosphate substrates, including S1P, ceramide-1-phosphate, lysophosphatidic acid, and phosphatidic acid. ${ }^{46}$ However, S1P lyase, an endoplasmic reticulum-bound enzyme, irreversibly degrades S1P and functions along with SphK1 as the main regulator of steadystate intracellular S1P levels, as shown by the impact of its inactivation in cells, tissues, and organisms. ${ }^{15}$

\section{Sphingosine kinase I}

SphK1 is the primary enzyme responsible for S1P generation and is mainly localized in the cytosol. It is activated by diverse stimuli such as growth factors, including epidermal growth factor (EGF), insulin-like growth factor-1, hormones (estradiol), and the angiogenic factor vascular endothelial 


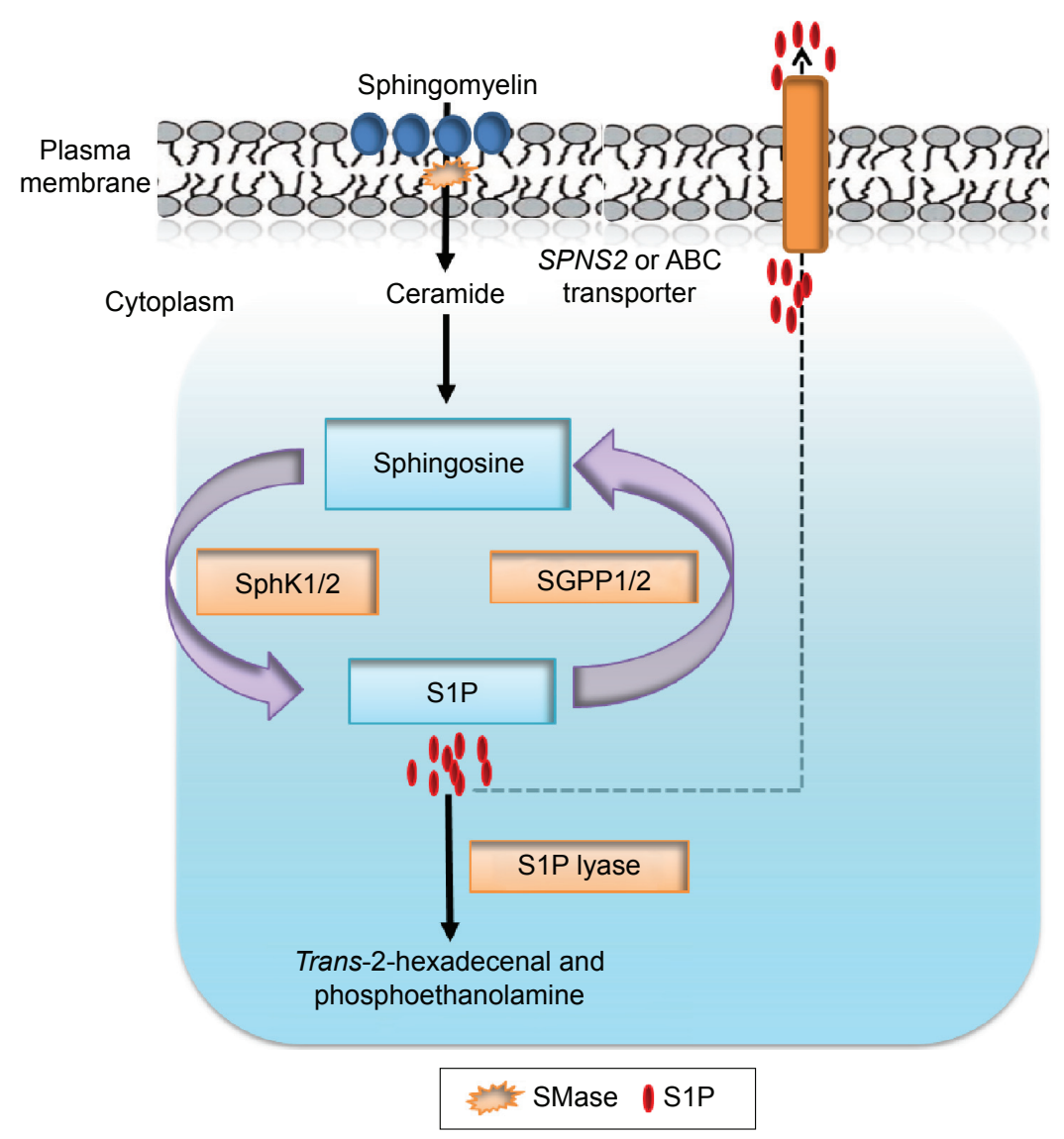

Figure I SIP metabolic pathway.

Notes: SMase catalyzes the hydrolysis of sphingomyelin to produce ceramide. Ceramide is further catabolized by ceramidase to generate sphingosine. SIP is produced by phosphorylation of sphingosine in a reaction catalyzed by SphKs. SIP is exported out through SPNS2 or ABC transporter. Intracellular concentration of SIP is regulated by the balance between its synthesis and further metabolism by (SGPP) and SIP lyase, which degrades SIP into hexadecenal and phosphoethanolamine.

Abbreviations: SIP, sphingosine-I-phosphate; SMase, sphingomyelinase; SphKs, sphingosine kinases; SPNS2, spinster homolog 2; ABC, ATP-binding cassette; SGPP, SIP phosphatase.

growth factor. ${ }^{47}$ Upon stimulation by a wide variety of growth factors, cytokines, and lipopolysaccharides, SphK1 gets phosphorylated at ser225 and translocates to the plasma membrane, resulting in its enhanced catalytic activity. ${ }^{48}$ SphK1 is an established oncogene, and it is overexpressed in various types of human cancers, including prostate, gastric, breast, lung, colon, glioma, Hodgkin's lymphoma, and HNSCC. ${ }^{14,49}$ Pharmacological inhibition of SphK1 enzyme activity or Sphk1 gene silencing blocks tumor progression in many types of cancers. ${ }^{14}$ Several recent reports suggest that SphK1 plays an important role in HNSCC carcinogenesis, invasion, metastasis, and radiosensitization. ${ }^{49-52}$

SphK1 expression levels were significantly higher in OSCC primary tumors, ${ }^{50,51,53}$ squamous carcinoma cell lines, and lymph nodes. ${ }^{50}$ Moreover, OSCC patients exhibiting strong immunostaining for SphK1 had shorter survival times. ${ }^{53}$ In a chemical-induced model of tongue cancer, SphK1-knockout mice exhibited decreased tumor incidence, multiplicity, and volume when compared with wild-type mice, suggesting that SphK1 is necessary for HNSCC carcinogenesis. ${ }^{51}$ SphK1 has also been implicated in regulating tumor invasion and metastasis in squamous cell carcinoma of tongue ${ }^{49}$ and esophagus. ${ }^{54}$ Epithelialmesenchymal transition (EMT) and degradation of the extracellular matrix are early events in metastasis. Matrix metalloproteinases (MMP) play a critical role in ECM degradation and remodeling processes that are required for EMT. Loss of E-cadherin is considered to be a fundamental event in EMT, and E-cadherin expression is inversely correlated with lymph node metastasis in primary HNSCC. Indeed, SphK1 overexpression in tongue squamous carcinoma cells (SCC-25) was associated with increased levels of MMP-2, MMP-9, EGF, and Akt activity and decreased level of E-cadherin. The STAT3 signaling pathway and the S1P/ S1PR1 pathway coactivate one another in an inflammatory loop that promotes carcinogenesis. Notably, SphK1 overexpression in SCC-25 cells also increases interleukin 6 (IL-6) concentration and EGF-induced STAT3 phosphorylation in 
S1PR1-dependent manner, suggesting that SphK1 modulates IL-6/STAT3 signaling during invasion. ${ }^{49}$

EGFR overexpression and chromosome $3 p$ deletion are two frequent events in HNSCC. LRIG1, a negative regulator of EGFR, is located at 3p14 and its copy-number loss correlates with poor clinical outcome. Activation of EGFR-mitogen-activated protein kinases (MAPK) signaling increases SphK1 activity, both increasing its expression level and promoting its phosphorylation. ${ }^{48}$ Interestingly, S1P has been shown to induce EGFR transactivation through S1PRs. ${ }^{55}$ Notably, LRIG overexpression in nasopharyngeal carcinoma attenuates EGFR-MAPK signaling and decreases SphK1 expression. ${ }^{52}$ Conversely, downregulation of LRIG1 in cancer cells enhances EGFR-MAPK-SphK1 signaling and ECM remodeling activity, leading to malignant phenotype of HNC. ${ }^{52}$

Development of resistance to targeted therapeutic drugs in HNSCC is a major therapeutic challenge. SphK1 plays a crucial role in cancer drug resistance and radioresistance. It has been proposed that release of S1P into the tumor microenvironment due to upregulation of SphK1 could represent an important mechanism of resistance. ${ }^{56,57}$ Recently, it has been shown that E2F7 transcription factor contributes to anthracycline resistance in HNSCC cells in a SphK1-dependent manner. E2F7 directly induces the expression of SphK1 in squamous carcinoma cells resulting in increased Akt activation, which drives drug resistance. ${ }^{58}$ SKI-II, a pharmacological inhibitor of SphK1, and short interfering RNA (siRNA)-mediated SphK1 silencing restore radiation sensitivity in radiation-resistant tongue squamous carcinoma cells (SCC-15 and SCC-25).${ }^{50}$ Further, SCC-15 cells transfected with SphK1-siRNA produce smaller tumors in mice compared to parent cells. ${ }^{50}$ SKI-II along with the EGFR inhibitor cetuximab reduced clonogenic survival in radiation-resistant HNSCC cells. ${ }^{59}$ Furthermore, a doxorubicin and SphK1 inhibitor SK1-I reduced tumor size in xenografted mice by sensitizing the SCC cells, which were resistant to doxorubicin alone. ${ }^{58}$ The above studies suggest that targeting SphK1/S1P might provide a novel strategy for the treatment of advanced stages of HNSCC that are resistant to EGFR therapy.

\section{Sphingosine kinase 2}

While SphK1 has been the subject of intense investigation, SphK2 has not received as much attention. Early studies showed that SphK2 can oppose some functions of SphK1. For example, SphK2 has been shown to induce apoptosis, whereas SphK1 suppresses apoptosis. ${ }^{60-62} \mathrm{SphK} 2$ is localized in the mitochondria, nucleus, and endoplasmic reticulum. $\mathrm{S} 1 \mathrm{P}$ produced in the nucleus by SphK2 inhibits HDAC1/2 activity similar to classical HDAC inhibitors, whereas reduction of nuclear S1P increases HDAC activity and concomitantly decreases histone acetylation. ${ }^{34} \mathrm{SphK} 2$ has shown a protective role in the development of colitisassociated cancer (CAC) in mice, whereas SphK2 deficiency exacerbates CAC. ${ }^{17}$ Interestingly, it has been shown that nuclear S1P produced by nuclear SphK2 suppresses transcription of SphK1 by inhibiting HDAC1/2 and activating the transcription factors c-Jun and AP-1. Indeed, Sphk2deficient mice exhibit increased levels of SphK1 mRNA and protein and elevated levels of plasma S1P. ${ }^{17}$ In contrast, SphK2 has also been shown to play a crucial role in tumor progression in several types of cancers, ${ }^{61}$ including multiple myeloma, ${ }^{62}$ lymphoblastic leukemia, ${ }^{63}$ prostate cancer, ${ }^{64}$ lung cancer, ${ }^{65}$ and breast cancer. ${ }^{66}$ However, the potential of SphK2 as a therapeutic target in cancer remains unclear and in HNSCC has not been explored at all. Nonetheless, few selective inhibitors of SphK2 have shown antitumor, antiangiogenic activities in cancer cell lines as well as mouse models. ${ }^{67-69}$

\section{SIP phosphatases}

SGPP1 is expressed in most tissues, with the highest levels found in the vascular tissues of placenta and kidney, ${ }^{45}$ whereas SGPP2 is predominantly expressed in the heart and kidney. ${ }^{70}$ In mammalian cells, overexpression of SGPP1 elevates ceramide levels and enhances apoptosis in cancer cells. SGPP1 regulates EGF-induced chemotaxis, ${ }^{44}$ autophagy, ${ }^{71}$ and keratinocyte differentiation. ${ }^{72}$ These observations suggest that it might play an important role in squamous cell carcinoma, as all these processes have been linked to HNSCC carcinogenesis. SGPP1 has been associated with drug resistance in ovarian cancer. ${ }^{73}$ The expression of SGPP2 is decreased in gliomas compared to normal gray matter, resulting in the accumulation of S1P in the tumors. ${ }^{74}$ SGPP1 has been identified as a target gene for oncogenic micro-RNA (miR)-21 and miR-95 in cancer stem-like cells isolated from lung cancer patients. ${ }^{75}$ The elevated expression of miR-21 and miR-95 contributes to radioresistance in lung, prostate, and breast cancers by decreasing the protein levels of SGPP1. ${ }^{75,76}$ In contrast, SGPP1 has also been identified as a target gene of tumor suppressor miR-27a in human colorectal tumors and colorectal cancer cell lines. ${ }^{76}$ SGPP2 has been identified as a novel vitamin D-responsive gene associated with lung function. ${ }^{77}$ However, the role of S1P phosphatases in HNSCC has not yet been explored. 


\section{Lipid phosphate phosphatases}

Expression of LPP1 is low in many cancer cell lines and tumors compared with normal tissues. Overexpression of LPP1 in cancer cells attenuates tumor growth and metastasis in mice. ${ }^{78}$ LPP 3 expression decreases the growth, survival, and tumorigenesis of ovarian cancer cells. ${ }^{79}$ In contrast, elevated expression of LPP3 was observed in primary glioblastoma tumors and in U87 and U118 glioblastoma cell lines. Further, LPP3 knockdown inhibits both glioblastoma cell proliferation in culture and tumor growth in xenograft assays by reducing $\beta$-catenin, cyclin D1, and CD133 expression. ${ }^{80}$

\section{SIP lyase}

S1P lyase expression is downregulated in human colon adenomas and prostate adenocarcinoma tissues. ${ }^{81,82}$ Furthermore, S1P lyase deficiency in human cancer cells induces resistance to chemotherapeutic drugs and ionizing radiation. ${ }^{43,81,82}$ In a CAC model, mice lacking intestinal S1P lyase exhibited greater disease activity, colon shortening, cytokine levels, S1P accumulation, increased tumor incidence, STAT3 activation, STAT3-activated miRs (miR-21 and miR-181b-1), and suppression of miR-targeted antioncogene products (PTEN and CYLD). ${ }^{83}$ Indeed, HPV-positive HNSCC tumors exhibit frequent genetic alterations in $P T E N{ }^{84}$ Recently, it has been shown that upregulation of ATP-binding cassette (ABC) transporters contributes to chemoresistance of S1P lyasedeficient fibroblasts. ${ }^{56}$ Whether S1P catabolizing enzymes play any role in HNSCC needs further study.

\section{SIP transporters}

$\mathrm{S} 1 \mathrm{P}$ is generated intracellularly by SphKs, and it is exported out of the cells by transporter proteins. Several $A B C$ family members, such as ABCA1, ABCC1, and ABCG1, have been proposed to transport S1P. ${ }^{56}$ Spinster homolog 2 (Spns2) is a non-ATP-dependent S1P transporter. ${ }^{57,85,86}$ Advanced lung cancer patients exhibit decreased Spns 2 expression, and ectopic Spns 2 expression induced apoptosis in non-smallcell lung cancer cells, whereas its knockdown enhanced cell migration. ${ }^{87}$

\section{SIPRs in HNSCC}

Many functions of S1P have been attributed to the activation of S1PRs. ${ }^{12,88,89}$ To date, five S1PRs (S1PR1, S1PR2, S1PR3, S1PR4 and S1PR5) have been identified in vertebrates. S1PRs are ubiquitously but differentially expressed on all cells. ${ }^{90}$ Each S1PR couples to different heterotrimeric G-proteins and activates or inhibits downstream signaling pathways (Figure 2). For example, S1PR1 and S1PR4 couple mainly to $G_{i}$, whereas S1PR2 and S1PR3 activate $G_{i}$,
$\mathrm{G}_{\mathrm{q}}$ and $\mathrm{G}_{12 / 13}$. S1PR5 binds to $\mathrm{G}_{\mathrm{i}}$ and $\mathrm{G}_{12 / 13} \cdot{ }^{91,92}$ Downstream signaling pathways that are activated or inhibited by S1PRs include extracellular signal-regulated kinase (ERK), c-Jun N-terminal kinase, PI3K, Rac, Rho, cyclic adenosine monophosphate, and phospholipase C. ${ }^{92}$

While S1PRs have been specifically studied in different types of cancer such as breast, ${ }^{93}$ gastric, ${ }^{94}$ thyroid, ${ }^{95,96}$ melanoma, ${ }^{97}$ and glioblastoma, ${ }^{98,99}$ their role in HNSCC is poorly understood. STAT3 is a key transcription factor implicated in inflammation and cancer. ${ }^{100}$ STAT3 is activated by many growth factors and inflammatory cytokines, including IL-6. S1P signaling through S1PR1 participates in a reciprocal positive feedback loop with STAT3 resulting in latter's persistent activation ${ }^{101}$ and promotes tumor progression and metastasis. ${ }^{83,101}$ S1P-S1PR1-STAT3 feedback loop plays an essential role in tumorigenesis of colorectal cancer, ${ }^{17,83}$ neuroblastoma, ${ }^{102}$ and HNSCC .99 S1P mediates invasion of HNSCC cells through S1PR1, and pharmacological inhibition or genetic knockdown of S1PR1 gene reduces invasion of SCC-25 cells through an IL-6/ STAT3-mediated pathway. ${ }^{49}$

S1PR2 activation has been shown to inhibit cancer cell migration, invasion, and metastasis. ${ }^{103,104}$ Crosstalk between S1P-S1PR2 and transforming growth factor beta signaling pathways has been implicated in progression and invasion of esophageal cancer through ERK1/2 activation. ${ }^{105}$ Targeted disruption of the SIPR 2 in mice leads to diffuse large B-cell lymphoma formation, ${ }^{106}$ and mutations in $S 1 P R 2$ gene has been reported in germinal center B-cell like diffuse large B-cell lymphoma. ${ }^{107}$ Whether similar mutations occur in SIPR2 gene in HNSCC is not yet known.

One theory of carcinogenesis espouses that cancers arise from and are perpetuated by a subpopulation of tumor cells called cancer stem cells (CSCs). CSCs exhibit radiation and chemotherapy-resistant properties and can give rise to all other cells in tumors from which they are isolated. S1P has been shown to play a fundamental role in the expansion of glioma stem-like cells ${ }^{108}$ and CSCs through the activation of S1PR3. ${ }^{109}$ High expression of S1PR4 in estrogen receptornegative breast cancer patients is associated with shorter disease-free and disease-specific survival. ${ }^{110}$ S1PR5 has been shown to exert anticancer effects by inducing autophagy in PC3 prostate cancer cells ${ }^{111}$ and by inhibiting migration and proliferation in esophageal cancer cells. ${ }^{112}$

\section{Therapeutic approaches for HNSCC targeting SIP signaling pathway}

Owing to the central role of S1P signaling in immunity and tumorigenesis and the druggability of SphKs and S1PRs, S1P 


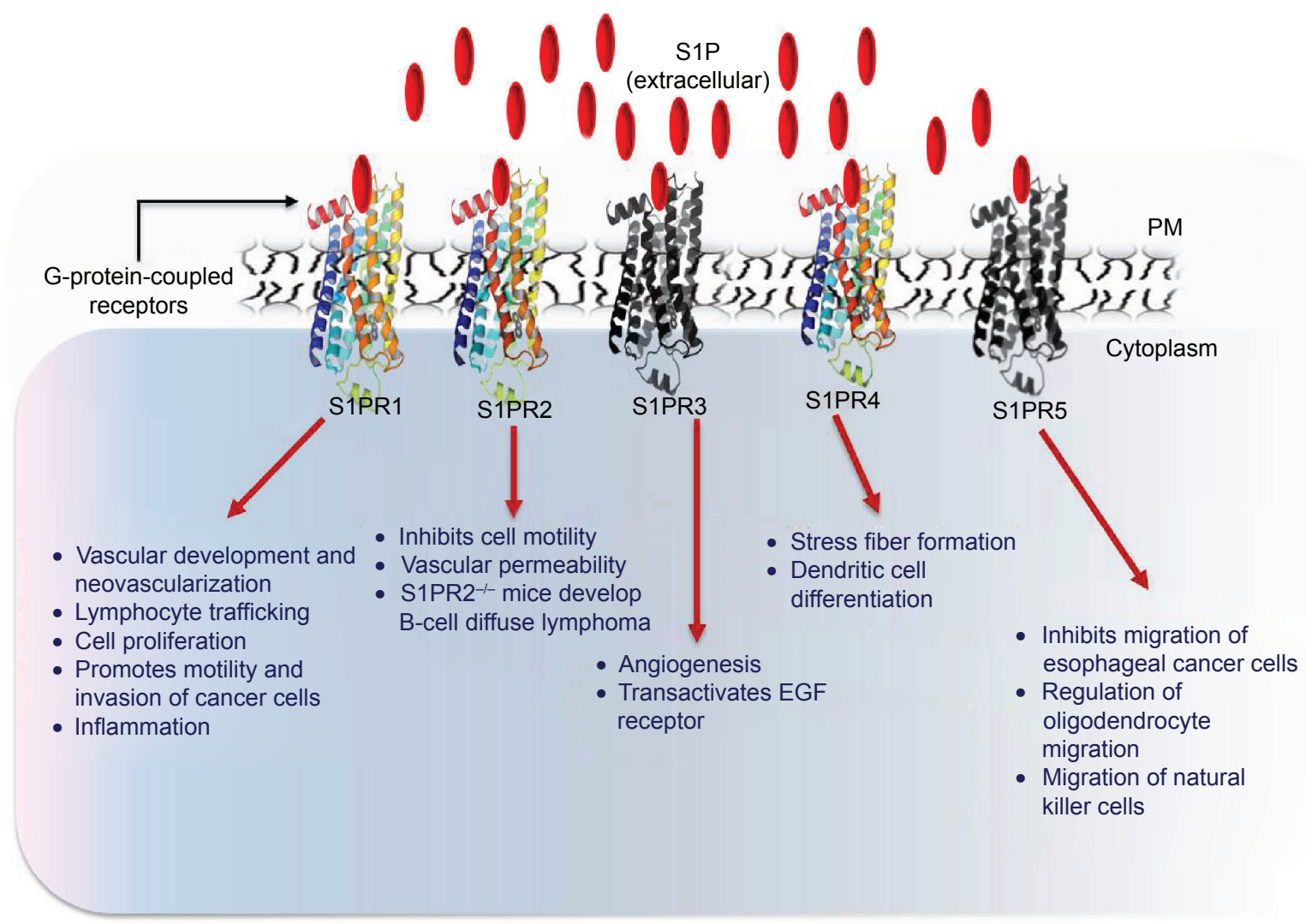

Figure 2 SIP mediates its functions through SIPRs.

Notes: Extracellular SIPs engage with SIPRs (I-5) that activate a number of signaling molecules, including ERKI/2, PLC, and small GTPase of the Rho family. SIP-SIPR signaling regulates myriad of cellular and physiological processes.

Abbreviations: SIP, sphingosine-I-phosphate; SIPR, SIP receptor; ERK, extracellular signal-regulated kinase; PLC, phospholipase C; EGF, epidermal growth factor; PM, plasma membrane.

signaling pathway is an attractive pharmacological target for drug discovery (Figure 3). Cumulative evidence from studies of many types of cancers suggests the involvement of SphK1 in tumor progression, invasion, metastasis, and radio- and chemoresistance. Furthermore, high expression of SphK1 correlates with poor patient outcomes in many cancers, including HNSCC. Selective SphK1 inhibitors have been tested in cell lines and animal models of several types of cancers, ${ }^{113} \mathrm{eg}$, leukemia ${ }^{114}$ and glioblastoma. ${ }^{115}$ Safingol, a SphK1 inhibitor combined with cisplatin,

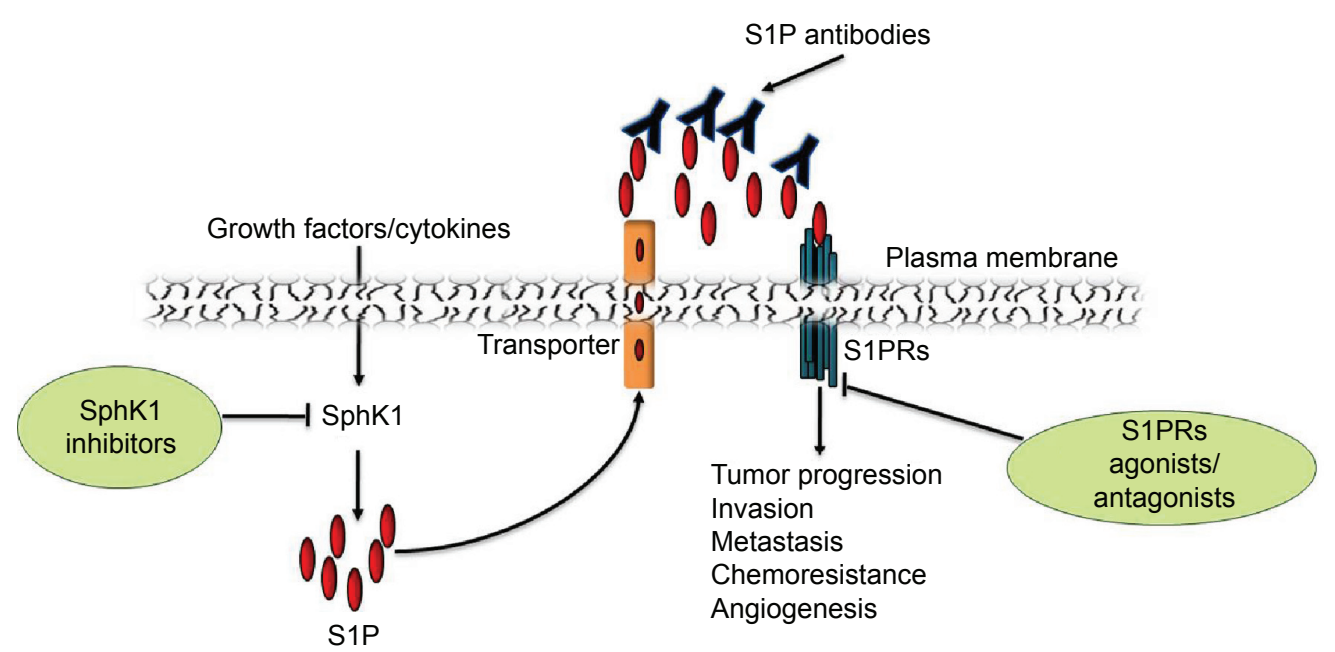

Figure 3 SphK activity is increased in response to various stimuli such as growth factors, which results in intracellular generation of SIP.

Notes: After its release in the extracellular space, SIP can activate its receptors and promotes tumor progression, angiogenesis, invasion, and metastasis of HNSCC. Various components of SIP signaling pathway, such as SIP, SphK, and SIPRs, are potential drug targets for cancer.

Abbreviations: SphK, sphingosine kinase; SIP, sphingosine-I-phosphate; HNSCC, head and neck squamous cell carcinoma; SIPRs, SIP receptors. 
has been successfully tested in a Phase I clinical trial for advanced solid tumors. ${ }^{116}$ Safingol induces apoptosis in OSCC by generating reactive oxygen species. ${ }^{117}$ In addition to SphK1 inhibitors, ABC294640, a SphK2 selective inhibitor, suppresses cell proliferation of a number of cancer cell lines and reduces the in vivo tumor growth of mammary adenocarcinoma xenografts. ${ }^{66}$ ABC294640 also exhibits synergistic effects with sorafenib, a multikinase inhibitor. ${ }^{118}$ Gold nanorods (GNRs) play a critical role in protecting the siRNA molecules against physiological degradation as well as delivering them inside target cells. SphK1-siRNA delivered as a GNR-siRNA complex demonstrate enhanced radiosensitization of HNSCC cells in vitro. ${ }^{119}$ Furthermore, intratumor injection of GNR-siRNA nanoplexes in HNSCC tumors grown in mice, prior to the initiation of radiation therapy, results in $>50 \%$ tumor regression compared to that of control. ${ }^{119}$ Sphingomab, a murine monoclonal S1P antibody, acts as "molecular sponge" to neutralize plasma S1P. ${ }^{120}$ It has been shown to reduce cell growth, invasion, and angiogenesis in multiple tumor lineages, including ovarian, lung, and breast cancer. ${ }^{121}$ Sonepcizumab, ${ }^{122,123}$ a humanized version of sphingomab, was developed and tested for safety and dosage tolerability in Phase I clinical trials for solid tumors. ${ }^{123}$ Though a Phase II clinical trial (NCT01762033) of sonepcizumab in renal cancer (late stage disease) conducted by LPath, Inc. (San Diego, CA, USA) has been unsuccessful, the Phase II trial has not been done with HNSCC.

A number of therapeutic agents that target the sphingolipid pathway have been developed. ${ }^{124,125}$ FTY720, a synthetic analog of sphingosine, which binds to all the S1PRs, except S1PR2, has been approved by the US Food and Drug Administration for treatment of patients with the autoimmune disease relapsing multiple sclerosis patients. FTY720 inhibits tumor development and angiogenesis in mice harboring human hepatocellular, bladder, and lung cancers. ${ }^{126,127}$ Therefore, there is potential for repurposing this US Food and Drug Administration approved drug for treatment of cancer patients. Several S1PR-specific agonist and antagonist compounds have been generated for therapeutic purposes. A more specific and potent agonist of S1PR1, siponimod (also known as BAF312), has been developed, which is now used in Phase III trials for multiple sclerosis. ${ }^{30}$ Whether these agonist or antagonist compounds could be used as a drug in HNSCC needs further investigation. A number of obstacles must be overcome if targeting S1PRs for cancer treatment is to be successful. Not all S1PRs mediate tumorigenic responses; hence, selectivity of receptor antagonism is crucial in determining the success of sphingolipid receptor-based therapeutics. ${ }^{14,30,124}$

\section{Conclusion}

$\mathrm{S} 1 \mathrm{P}$ signaling regulates many aspects of $\mathrm{HNSCC}$ carcinogenesis, and S1P-targeted therapy for HNSCC is an exciting possibility. SphK1 inhibitors in combination with conventional chemotherapy agents, such as doxorubicin and cisplatin, have been used for sensitizing squamous carcinoma cells that were either resistant to radiation, doxorubicin, or cisplatin alone. Until now, most studies have focused on SphK1. However, the potential of targeting other S1P-related proteins, such as S1P catabolizing enzymes, transporters, and S1PRs, in HNSCC has yet to be investigated. Furthermore, novel strategies that combine targeting both the S1P signaling pathway and other carcinogenic pathways with which it interacts (especially those implicated in HNSCC), such as EGFR, STAT3, AKT, ERK/MAPK, and NF- $\mathrm{BB}$, could be very effective. For example, a Phase II clinical trial using the S1P antibody sonepcizumab for cancer treatment has been initiated and might represent a new potential therapeutic option for HNSCC in combination with EGFR inhibitors. ${ }^{123}$ To overcome the problem of radioresistance, actively targeted gold nanoparticles have been tested as novel radiosensitizer agents in HNSCC. GNRs carrying SphK1-siRNA have shown promising results in mouse models for radiosensitizing HNSCC tumors. Thus, identification of potent and specific inhibitors of SK1 and selective agonist/antagonist of S1PRs combined with novel drug delivery tools may improve the prognosis of HNSCC patients. As we learn more about this interesting growth-regulatory signaling pathway, its potential to serve as a useful therapeutic target and biomarker of disease in HNSCC will be revealed.

\section{Acknowledgments}

This work was supported by the Science and Engineering Research Board, Government of India's Scheme for Young Scientist research grant to Ashok Kumar (SB/YS/ LS-184/2013). Rajeev Nema is a recipient of a Research Associate Fellowship from the Indian Council of Medical Research (number 3/2/2/234/2014/NCD-III). The authors thank Doctor Julie D Saba and Doctor Rohit Saluja for critical reading of the manuscript.

\section{Disclosure}

The authors report no conflicts of interest in this work.

\section{References}

1. Kang H, Kiess A, Chung CH. Emerging biomarkers in head and neck cancer in the era of genomics. Nat Rev Clin Oncol. 2015;12(1):11-26.

2. Parkin DM, Bray F, Ferlay J, Pisani P. Global cancer statistics, 2002. CA Cancer J Clin. 2005;55(2):74-108. 
3. Amusa YB, Olabanji JK, Akinpelu VO, et al. Pattern of head and neck malignant tumors in a Nigerian teaching hospital - a ten year review. West Afr J Med. 2004;23(4):280-285.

4. Adeyemi BF, Kolude BM, Ogun GO, Akang EE. Paediatric head and neck malignancies in Ibadan, Nigeria. Afr J Med Med Sci. 2009;38(1):55-62.

5. Mallath MK, Taylor DG, Badwe RA, et al. The growing burden of cancer in India: epidemiology and social context. Lancet Oncol. 2014; 15(6):e205-e212.

6. Fung C, Grandis JR. Emerging drugs to treat squamous cell carcinomas of the head and neck. Expert Opin Emerg Drugs. 2010;15(3):355-373.

7. Vincenzi B. Hot topic: biology in anticancer treatment. Curr Cancer Drug Targets. 2010;10(1):1-2.

8. Guigay J, Fayette J, Dillies AF, et al. Cetuximab, docetaxel and cisplatin as first-line treatment in patients with recurrent or metastatic head and neck squamous cell carcinoma: a multicentre, phase II GORTEC study. Ann Oncol. 2015;26(9):1941-1947.

9. Martinez-Useros J, Garcia-Foncillas J. The challenge of blocking a wider family members of EGFR against head and neck squamous cell carcinomas. Oral Oncol. 2015;51(5):423-430.

10. Brand TM, Iida M, Wheeler DL. Molecular mechanisms of resistance to the EGFR monoclonal antibody cetuximab. Cancer Biol Ther. 2011; 11(9):777-792.

11. Cohen RB. Current challenges and clinical investigations of epidermal growth factor receptor (EGFR)- and ErbB family-targeted agents in the treatment of head and neck squamous cell carcinoma (HNSCC). Cancer Treat Rev. 2014;40(4):567-577.

12. Spiegel S, Milstien S. Sphingosine-1-phosphate: an enigmatic signalling lipid. Nat Rev Mol Cell Biol. 2003;4(5):397-407.

13. Fyrst H, Saba JD. An update on sphingosine-1-phosphate and other sphingolipid mediators. Nat Chem Biol. 2010;6(7):489-497.

14. Pyne NJ, Pyne S. Sphingosine 1-phosphate and cancer. Nat Rev Cancer. 2010;10(7):489-503.

15. Kumar A, Saba JD. Lyase to live by: sphingosine phosphate lyase as a therapeutic target. Expert OpinTher Targets. 2009;13(8):1013-1025.

16. Ogretmen B, Hannun YA. Biologically active sphingolipids in cancer pathogenesis and treatment. Nat Rev Cancer. 2004;4(8):604-616.

17. Liang J, Nagahashi M, Kim EY, et al. Sphingosine-1-phosphate links persistent STAT3 activation, chronic intestinal inflammation, and development of colitis-associated cancer. Cancer Cell. 2013;23(1):107-120.

18. Feller LL, Khammissa RR, Kramer BB, Lemmer JJ. Oral squamous cell carcinoma in relation to field pre-cancerisation: pathobiology. Cancer Cell Int. 2013;13(1):31.

19. Pai SI, Westra WH. Molecular pathology of head and neck cancer: implications for diagnosis, prognosis, and treatment. Annu Rev Pathol. 2009;4:49-70.

20. Leemans CR, Braakhuis BJ, Brakenhoff RH. The molecular biology of head and neck cancer. Nat Rev Cancer. 2011;11(1):9-22.

21. Stransky N, Egloff AM, Tward AD, et al. The mutational landscape of head and neck squamous cell carcinoma. Science. 2011 333(6046):1157-1160.

22. ZurHausen H. Papillomaviruses and cancer: from basic studies to clinical application. Nat Rev Cancer. 2002;2(5):342-350.

23. Nemunaitis J, Nemunaitis J. Head and neck cancer: response to p53based therapeutics. Head Neck. 2011;33(1):131-134.

24. Gross AM, Orosco RK, Shen JP, et al. Multi-tiered genomic analysis of head and neck cancer ties TP53 mutation to 3p loss. Nat Genet. 2014; 46(9):939-943.

25. Cassell A, Grandis JR. Investigational EGFR-targeted therapy in head and neck squamous cell carcinoma. Expert Opin Investig Drugs. 2010; 19(6):709-722.

26. Pickering CR, Zhang J, Yoo SY, et al. Integrative genomic characterization of oral squamous cell carcinoma identifies frequent somatic drivers. Cancer Discov. 2013;3(7):770-781.

27. Chang SS, Califano J. Current status of biomarkers in head and neck cancer. J Surg Oncol. 2008;97(8):640-643.

28. Tsui IF, Rosin MP, Zhang L, Ng RT, Lam WL. Multiple aberrations of chromosome $3 \mathrm{p}$ detected in oral premalignant lesions. Cancer Prev Res (Phila). 2008;1(6):424-429.
29. Rivera J, Proia RL, Olivera A. The alliance of sphingosine-1-phosphate and its receptors in immunity. Nat Rev Immunol. 2008;8(10):753-763.

30. Kunkel GT, Maceyka M, Milstien S, Spiegel S. Targeting the sphingosine-1-phosphate axis in cancer, inflammation and beyond. Nat Rev Drug Discov. 2013;12(9):688-702.

31. Mandala S, Hajdu R, Bergstrom J, et al. Alteration of lymphocyte trafficking by sphingosine-1-phosphate receptor agonists. Science. 2002; 296(5566):346-349.

32. Kihara Y, Mizuno H, Chun J. Lysophospholipid receptors in drug discovery. Exp Cell Res. 2015;333(2):171-177.

33. Alvarez SE, Harikumar KB, Hait NC, et al. Sphingosine-1-phosphate is a missing cofactor for the E3 ubiquitin ligase TRAF2. Nature. 2010; 465(7301):1084-1088.

34. Hait NC, Allegood J, Maceyka M, et al. Regulation of histone acetylation in the nucleus by sphingosine-1-phosphate. Science. 2009;325(5945): 1254-1257.

35. Takasugi N, Sasaki T, Suzuki K, et al. BACE1 activity is modulated by cell-associated sphingosine-1-phosphate. J Neurosci. 2011;31(18): 6850-6857.

36. Kumar A, Saba DJ. Sphingosine-1-phosphate. In: Choi S, editor. Encyclopedia of Signaling Molecules. New York, NY: Springer; 2012: 1771.

37. Argraves KM, Argraves WS. HDL serves as a S1P signaling platform mediating a multitude of cardiovascular effects. J Lipid Res. 2007;48(11): 2325-2333.

38. Christoffersen C, Obinata H, Kumaraswamy SB, et al. Endotheliumprotective sphingosine-1-phosphate provided by HDL-associated apolipoprotein M. Proc Natl Acad Sci U S A. 2011;108(23):9613-9618.

39. Blaho VA, Hla T. An update on the biology of sphingosine 1-phosphate receptors. J Lipid Res. 2014;55(8):1596-1608.

40. Sutphen R, Xu Y, Wilbanks GD, et al. Lysophospholipids are potential biomarkers of ovarian cancer. Cancer Epidemiol Biomarkers Prev. 2004;13(7):1185-1191.

41. Alberg AJ, Armeson K, Pierce JS, et al. Plasma sphingolipids and lung cancer: a population-based, nested case-control study. Cancer Epidemiol Biomarkers Prev. 2013;22(8):1374-1382.

42. Nunes J, Naymark M, Sauer L, et al. Circulating sphingosine-1-phosphate and erythrocyte sphingosine kinase-1 activity as novel biomarkers for early prostate cancer detection. Br J Cancer. 2012;106(5):909-915.

43. Kumar A, Oskouian B, Fyrst H, Zhang M, Paris F, Saba JD. S1P lyase regulates DNA damage responses through a novel sphingolipid feedback mechanism. Cell Death Dis. 2011;10(2):e119.

44. Le Stunff H, Mikami A, Giussani P, et al. Role of sphingosine1-phosphate phosphatase 1 in epidermal growth factor-induced chemotaxis. J Biol Chem. 2004;279(33):34290-34297.

45. Johnson KR, Johnson KY, Becker KP, Bielawski J, Mao C, Obeid LM Role of human sphingosine-1-phosphate phosphatase 1 in the regulation of intra- and extracellular sphingosine-1-phosphate levels and cell viability. J Biol Chem. 2003;278(36):34541-34547.

46. Brindley DN, Pilquil C. Lipid phosphate phosphatases and signaling. $J$ Lipid Res. 2009;50(suppl):S225-S230.

47. Alvarez SE, Milstien S, Spiegel S. Autocrine and paracrine roles of sphingosine-1-phosphate. Trends Endocrinol Metab. 2007;18(8):300-307.

48. Pitson SM, Powell JA, Bonder CS. Regulation of sphingosine kinase in hematological malignancies and other cancers. Anticancer Agents Med Chem. 2011;11(9):799-809.

49. Tamashiro PM, Furuya H, Shimizu Y, Kawamori T. Sphingosine kinase 1 mediates head \& neck squamous cell carcinoma invasion through sphingosine 1-phosphate receptor 1. Cancer Cell Int. 2014;14(1):76.

50. Sinha UK, Schorn VJ, Hochstim C, Chinn SB, Zhu S, Masood R. Increased radiation sensitivity of head and neck squamous cell carcinoma with sphingosine kinase 1 inhibition. Head Neck. 2011;33(2):178-188.

51. Shirai K, Kaneshiro T, Wada M, et al. A role of sphingosine kinase 1 in head and neck carcinogenesis. Cancer Prev Res (Phila). 2011;4(3):454 462.

52. Sheu JJ, Lee CC, Hua CH, et al. LRIG1 modulates aggressiveness of head and neck cancers by regulating EGFR-MAPK-SPHK1 signaling and extracellular matrix remodeling. Oncogene. 2014;33(11):1375-1384. 
53. Facchinetti MM, Gandini NA, Fermento ME, et al. The expression of sphingosine kinase-1 in head and neck carcinoma. Cells Tissues Organs. 2010;192(5):314-324.

54. Pan J, Tao YF, Zhou Z, et al. An novel role of sphingosine kinase-1 (SPHK1) in the invasion and metastasis of esophageal carcinoma. $J$ Transl Med. 2011;9:157.

55. Tanimoto T, Lungu AO, Berk BC. Sphingosine 1-phosphate transactivates the platelet-derived growth factor beta receptor and epidermal growth factor receptor in vascular smooth muscle cells. Circ Res. 2004; 94(8):1050-1058.

56. Ihlefeld K, Vienken H, Claas RF, et al. Upregulation of ABC transporters contributes to chemoresistance of sphingosine 1-phosphate lyase-deficient fibroblasts. J Lipid Res. 2015;56(1):60-69.

57. Nagahashi M, Takabe K, Terracina KP, et al. Sphingosine-1-phosphate transporters as targets for cancer therapy. Biomed Res Int. 2014;2014: 651727.

58. Hazar-Rethinam M, de Long LM, Gannon OM, et al. A novel E2F/ sphingosine kinase 1 axis regulates anthracycline response in squamous cell carcinoma. Clin Cancer Res. 2015;21(2):417-427.

59. Schiefler C, Piontek G, Doescher J, et al. Inhibition of SphK1 reduces radiation-induced migration and enhances sensitivity to cetuximab treatment by affecting the EGFR/SphK1 crosstalk. Oncotarget. 2014; 5(20):9877-9888.

60. Liu H, Toman RE, Goparaju SK, et al. Sphingosine kinase type 2 is a putative BH3-only protein that induces apoptosis. J Biol Chem. 2003; 278(41):40330-40336.

61. Neubauer HA, Pitson SM. Roles, regulation and inhibitors of sphingosine kinase 2. FEBS J. 2013;280(21):5317-5336.

62. Venkata JK, An N, Stuart R, et al. Inhibition of sphingosine kinase 2 downregulates the expression of c-Myc and Mcl-1 and induces apoptosis in multiple myeloma. Blood. 2014;124(12):1915-1925.

63. Wallington-Beddoe CT, Powell JA, Tong D, Pitson SM, Bradstock $\mathrm{KF}$, Bendall LJ. Sphingosine kinase 2 promotes acute lymphoblastic leukemia by enhancing MYC expression. Cancer Res. 2014;74(10): 2803-2815.

64. Gestaut MM, Antoon JW, Burow ME, Beckman BS. Inhibition of sphingosine kinase-2 ablates androgen resistant prostate cancer proliferation and survival. Pharmacol Rep. 2014;66(1):174-178.

65. Wang Q, Li J, Li G, et al. Prognostic significance of sphingosine kinase 2 expression in non-small cell lung cancer. Tumor Biol. 2014;35(1): 363-368.

66. Gao P, Smith CD. Ablation of sphingosine kinase-2 inhibits tumor cell proliferation and migration. Mol Cancer Res. 2011;9(11): 1509-1519.

67. French KJ, Zhuang Y, Maines LW, et al. Pharmacology and antitumor activity of ABC294640, a selective inhibitor of sphingosine kinase-2. J Pharmacol Exp Ther. 2010;333(1):129-139.

68. Liu K, Guo TL, Hait NC, et al. Biological characterization of 3-(2-aminoethyl)-5-[3-(4-butoxyl-phenyl)-propylidene]-thiazolidine-2,4-dione (K145) as a selective sphingosine kinase-2 inhibitor and anticancer agent. PLoS One. 2013;8(2):e56471.

69. White MD, Chan L, Antoon JW, Beckman BS. Targeting ovarian cancer and chemoresistance through selective inhibition of sphingosine kinase-2 with ABC294640. Anticancer Res. 2013;33(9): $3573-3579$

70. Ogawa C, Kihara A, Gokoh M, Igarashi Y. Identification and characterization of a novel human sphingosine-1-phosphate phosphohydrolase, hSPP2. J Biol Chem. 2003;278(2):1268-1272.

71. Lépine S, Allegood JC, Edmonds Y, Milstien S, Spiegel S. Autophagy induced by deficiency of sphingosine-1-phosphate phosphohydrolase 1 is switched to apoptosis by calpain-mediated autophagy-related gene 5 (Atg5) cleavage. J Biol Chem. 2011;286(52):44380-44390.

72. Allende ML, Sipe LM, Tuymetova G, Wilson-Henjum KL, Chen W, Proia RL. Sphingosine-1-phosphate phosphatase 1 regulates keratinocyte differentiation and epidermal homeostasis. J Biol Chem. 2013;288(25): 18381-18391.
73. Helleman J, Jansen MP, Span PN, et al. Molecular profiling of platinum resistant ovarian cancer. Int J Cancer. 2006;118(8):1963-1971.

74. Abuhusain HJ, Matin A, Qiao Q, et al. A metabolic shift favoring sphingosine 1-phosphate at the expense of ceramide controls glioblastoma angiogenesis. J Biol Chem. 2013;288(52):37355-37364.

75. Zhang J, Zhang C, Hu L, et al. Abnormal expression of miR-21 and miR-95 in cancer stem-like cells is associated with radioresistance of lung cancer. Cancer Invest. 2015;33(5):165-171.

76. Bao Y, Chen Z, Guo Y, et al. Tumor suppressor microRNA-27a in colorectal carcinogenesis and progression by targeting SGPP1 and Smad2. PLoS One. 2014;9(8):e105991.

77. Reardon BJ, Hansen JG, Crystal RG, et al. Vitamin D-responsive SGPP2 variants associated with lung cell expression and lung function. BMC Med Genet. 2013;14:122.

78. Tang X, Benesch MG, Dewald J, et al. Lipid phosphate phosphatase-1 expression in cancer cells attenuates tumor growth and metastasis in mice. J Lipid Res. 2014;55(11):2389-2400.

79. Tanyi JL, Morris AJ, Wolf JK, et al. The human lipid phosphate phosphatase-3 decreases the growth, survival, and tumorigenesis of ovarian cancer cells: validation of the lysophosphatidic acid signaling cascade as a target for therapy in ovarian cancer. Cancer Res. 2003;63(5): 1073-1082.

80. Chatterjee I, Humtsoe JO, Kohler EE, Sorio C, Wary KK. Lipid phosphate phosphatase-3 regulates tumor growth via $\beta$-catenin and CYCLIN-D1 signaling. Mol Cancer. 2011;10:51.

81. Oskouian B, Sooriyakumaran P, Borowsky AD, et al. Sphingosine1-phosphate lyase potentiates apoptosis via p53- and p38-dependent pathways and is down-regulated in colon cancer. Proc Natl Acad Sci US A. 2006;103(46):17384-17389.

82. Brizuela L, Ader I, Mazerolles C, Bocquet M, Malavaud B, Cuvillier O. First evidence of sphingosine 1-phosphate lyase protein expression and activity downregulation in human neoplasm: implication for resistance to therapeutics in prostate cancer. Mol Cancer Ther. 2012;11(9): 1841-1851.

83. Degagné E, Pandurangan A, Bandhuvula P, et al. Sphingosine-1phosphate lyase downregulation promotes colon carcinogenesis through STAT3-activated microRNAs. J Clin Invest. 2014;124(12): 5368-5384.

84. Chung CH, Guthrie VB, Masica DL, et al. Genomic alterations in head and neck squamous cell carcinoma determined by cancer gene-targeted sequencing. Ann Oncol. 2015;26(6):1216-1223.

85. Kawahara A, Nishi T, Hisano Y, Fukui H, Yamaguchi A, Mochizuki N. The sphingolipid transporter spns 2 functions in migration of zebrafish myocardial precursors. Science. 2009;323(5913):524-527.

86. Mendoza A, Bréart B, Ramos-Perez WD, et al. The transporter Spns 2 is required for secretion of lymph but not plasma sphingosine-1-phosphate. Cell Rep. 2012;2(5):1104-1110.

87. Bradley E, Dasgupta S, Jiang X, et al. Critical role of Spns2, a sphingosine-1-phosphate transporter, in lung cancer cell survival and migration. PLoS One. 2014;9(10):e110119.

88. Olivera A, Eisner C, Kitamura Y, et al. Sphingosine kinase 1 and sphingosine-1-phosphate receptor 2 are vital to recovery from anaphylactic shock in mice. J Clin Invest. 2010;20(5):1429-1440.

89. Saudemont A, Garçon F, Yadi H, et al. p110gamma and p1 10 delta isoforms of phosphoinositide 3-kinase differentially regulate natural killer cell migration in health and disease. Proc Natl Acad Sci U S A. 2009; 106(14):5795-5800.

90. Hla T, Brinkmann V. Sphingosine 1-phosphate (S1P): physiology and the effects of S1P receptor modulation. Neurology. 2011;76(8 suppl 3): S3-S8.

91. Takuwa Y, Takuwa N, Sugimoto N. The Edg family G protein-coupled receptors for lysophospholipids: their signaling properties and biological activities. J Biochem. 2002;131(6):767-771.

92. Taha TA, Argraves KM, Obeid LM. Sphingosine-1-phosphate receptors: receptor specificity versus functional redundancy. Biochim Biophys Acta. 2004;1682(1-3):48-55. 
93. Long JS, Fujiwara Y, Edwards J, et al. Sphingosine 1-phosphate receptor 4 uses HER2 (ERBB2) to regulate extracellular signal regulated kinase-1/2 in MDA-MB-453 breast cancer cells. $J$ Biol Chem. 2010;285(46):35957-35966.

94. Yamashita H, Kitayama J, Shida D, et al. Sphingosine 1-phosphate receptor expression profile in human gastric cancer cells: differential regulation on the migration and proliferation. J Surg Res. 2006;130(1): $80-87$.

95. Guan H, Liu L, Cai J, et al. Sphingosine kinase 1 is overexpressed and promotes proliferation in human thyroid cancer. Mol Endocrinol. 2011; 25(11):1858-1866.

96. Bergelin N, Löf C, Balthasar S, Kalhori V, Törnquist K. S1P1 and VEGFR-2 form a signaling complex with extracellularly regulated kinase $1 / 2$ and protein kinase C-alpha regulating ML-1 thyroid carcinoma cell migration. Endocrinology. 2010;151(7):2994-3005.

97. Arikawa K, Takuwa N, Yamaguchi H, et al. Ligand-dependent inhibition of B16 melanoma cell migration and invasion via endogenous S1P2 G protein-coupled receptor. Requirement of inhibition of cellular RAC activity. J Biol Chem. 2003;278(35):32841-32851.

98. Van Brocklyn JR, Young N, Roof R. Sphingosine-1-phosphate stimulates motility and invasiveness of human glioblastoma multiforme cells. Cancer Lett. 2003;199(1):53-60.

99. Brocklyn JR. Regulation of cancer cell migration and invasion by sphingosine-1-phosphate. World J Biol Chem. 2010;1(10):307-312.

100. Yu H, Pardoll D, Jove R. STATs in cancer inflammation and immunity: a leading role for STAT3. Nat Rev Cancer. 2009;9(11):798-809.

101. Lee H, Deng J, Kujawski M, et al. STAT3-induced S1PR1 expression is crucial for persistent STAT3 activation in tumors. Nat Med. 2010; 16(12):1421-1428.

102. Yang F, Jove V, Buettner R, et al. Sorafenib inhibits endogenous and IL-6/S1P induced JAK2-STAT3 signaling in human neuroblastoma, associated with growth suppression and apoptosis. Cancer Biol Ther. 2012;13(7):534-541.

103. Lepley D, Paik JH, Hla T, Ferrer F. The G protein-coupled receptor $\mathrm{S} 1 \mathrm{P} 2$ regulates $\mathrm{Rho} / \mathrm{Rho}$ kinase pathway to inhibit tumor cell migration. Cancer Res. 2005;65(9):3788-3795.

104. Young N, Van Brocklyn JR. Signal transduction of sphingosine-1phosphate G protein-coupled receptors. ScientificWorldJournal. 2006; 6:946-966.

105. Miller AV, Alvarez SE, Spiegel S, Lebman DA. Sphingosine kinases and sphingosine-1-phosphate are critical for transforming growth factor beta-induced extracellular signal-regulated kinase 1 and 2 activation and promotion of migration and invasion of esophageal cancer cells. Mol Cell Biol. 2008;28(12):4142-4151.

106. Cattoretti G, Mandelbaum J, Lee N, et al. Targeted disruption of the S1P2 sphingosine 1-phosphate receptor gene leads to diffuse large B-cell lymphoma formation. Cancer Res. 2009;69(22):8686-8692.

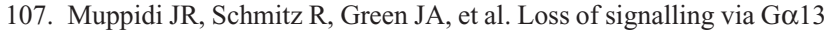
in germinal centre B-cell-derived lymphoma. Nature. 2014;516(7530): 254-258.

108. Marfia G, Campanella R, Navone SE, et al. Autocrine/paracrine sphingosine-1-phosphate fuels proliferative and stemness qualities of glioblastoma stem cells. Glia. 2014;62(12):1968-1981.

109. Hirata N, Yamada S, Shoda T, Kurihara M, Sekino Y, Kanda Y. Sphingosine-1-phosphate promotes expansion of cancer stem cells via S1PR3 by a ligand-independent Notch activation. Nat Commun. 2014;5: 4806.

110. Ohotski J, Long JS, Orange C, et al. Expression of sphingosine 1-phosphate receptor 4 and sphingosine kinase 1 is associated with outcome in oestrogen receptor-negative breast cancer. Br J Cancer. 2012; 106(8):1453-1459.
111. Chang CL, Ho MC, Lee PH, Hsu CY, Huang WP, Lee H. S1P(5) is required for sphingosine 1-phosphate-induced autophagy in human prostate cancer PC-3 cells. Am J Physiol Cell Physiol. 2009;297(2): C451-C458.

112. Hu WM, Li L, Jing BQ, et al. Effect of S1P5 on proliferation and migration of human esophageal cancer cells. World J Gastroenterol. 2010; 16(15):1859-1866.

113. Gao Y, Gao F, Chen K, Tian ML, Zhao DL. Sphingosine kinase 1 as an anticancer therapeutic target. Drug Des Devel Ther. 2015;9: 3239-3245.

114. Paugh SW, Paugh BS, Rahmani M, et al. A selective sphingosine kinase 1 inhibitor integrates multiple molecular therapeutic targets in human leukemia. Blood. 2008;112(4):1382-1391.

115. Kapitonov D, Allegood JC, Mitchell C, et al. Targeting sphingosine kinase 1 inhibits Akt signaling, induces apoptosis, and suppresses growth of human glioblastoma cells and xenografts. Cancer Res. 2009;69(17): 6915-6923

116. Dickson MA, Carvajal RD, Merrill AH Jr, Gonen M, Cane LM, Schwartz GK. A phase I clinical trial of safingol in combination with cisplatin in advanced solid tumors. Clin Cancer Res. 2011; 17(8):2484-2492.

117. Hamada M, Wakabayashi K, Masui A, Iwai S, Imai T, Yura Y. Involvement of hydrogen peroxide in safingol-induced endonuclease G-mediated apoptosis of squamous cell carcinoma cells. Int $J$ Mol Sci. 2014;15(2):2660-2671.

118. Beljanski V, Knaak C, Zhuang Y, Smith CD. Combined anticancer effects of sphingosine kinase inhibitors and sorafenib. Invest New Drugs. 2011;29(6):1132-1142.

119. Masood R, Roy I, Zu S, et al. Gold nanorod-sphingosine kinase siRNA nanocomplexes: a novel therapeutic tool for potent radiosensitization of head and neck cancer. Integr Biol (Camb). 2012;4(2): $132-141$

120. O'Brien N, Jones ST, Williams DG, et al. Production and characterization of monoclonal anti-sphingosine-1-phosphate antibodies. J Lipid Res. 2009;50(11):2245-2257.

121. Visentin B, Vekich JA, Sibbald BJ, et al. Validation of an antisphingosine-1-phosphate antibody as a potential therapeutic in reducing growth, invasion, and angiogenesis in multiple tumor lineages. Cancer Cell. 2006;9(3):225-238.

122. Lukowski ZL, Min J, Beattie AR, et al. Prevention of ocular scarring after glaucoma filtering surgery using the monoclonal antibody LT1009 (Sonepcizumab) in a rabbit model. J Glaucoma. 2013;22(2): 145-151.

123. Gordon MS, Just R, Rosen LS, Dorr L. A phase I study of sonepcizumab (S), a humanized monoclonal antibody to sphingosine-1-phosphate (S1P), in patients with advanced solid tumors. J Clin Oncol. 2010; 28:15S. [suppl; abstr 2560].

124. Proia RL, Hla T. Emerging biology of sphingosine-1-phosphate: its role in pathogenesis and therapy. J Clin Invest. 2015;125(4): 1379-1387.

125. Huwiler A, Zangemeister-Wittke U. Targeting the conversion of ceramide to sphingosine 1-phosphate as a novel strategy for cancer therapy. Crit Rev Oncol Hematol. 2007;63(2):150-159.

126. Ho JW, Man K, Sun CK, Lee TK, Poon RT, Fan ST. Effects of a novel immunomodulating agent, FTY720, on tumor growth and angiogenesis in hepatocellular carcinoma. Mol Cancer Ther. 2005; 4(9):1430-1438.

127. Salinas NR, Lopes CT, Palma PV, Oshima CT, Bueno V. Lung tumor development in the presence of sphingosine 1-phosphate agonist FTY720. Pathol Oncol Res. 2009;15(4):549-554. 


\section{Publish your work in this journal}

OncoTargets and Therapy is an international, peer-reviewed, open access journal focusing on the pathological basis of all cancers, potential targets for therapy and treatment protocols employed to improve the management of cancer patients. The journal also focuses on the impact of management programs and new therapeutic agents and protocols on

patient perspectives such as quality of life, adherence and satisfaction. The manuscript management system is completely online and includes a very quick and fair peer-review system, which is all easy to use. Visit http://www.dovepress.com/testimonials.php to read real quotes from published authors.

Submit your manuscript here: http://www.dovepress.com/oncotargets-and-therapy-journal 